\title{
Long-term Follow up of Two Cases with Spinal Hydatid Cyst Disease and Repeat Surgeries \\ $\mathrm{M} \mathrm{Kara}^{1}, \mathrm{AE} \mathrm{Baki}^{2}$, İ Batmaz ${ }^{3}$, FG Sarıkaya ${ }^{1}$, L Özçakar
}

\begin{abstract}
Hydatid cyst disease (HD) is an infestation caused by the larval stage of the tape worm Echinococcus granulosus. It predominantly occurs in liver and lungs. Bone involvement is quite rare $(0.5 \%-4 \%)$; and among those, spinal disease is the most common type (44\%-50\%). Reported here are two cases of spinal hydatid cyst disease in which neurogenic bladder and paraplegia due to spinal cord compression were encountered despite recurrent medical and surgical treatment. In this regard, we would like to emphasise that this-otherwise benigninfectious disease may pose significant challenges regarding musculoskeletal system and that early prompt management is paramount.
\end{abstract}

Keywords: Hydatid cyst, neurogenic bladder, paraplegia, spine, surgery

From: ${ }^{1}$ Department of Physical and Rehabilitation Medicine, Ankara Physical and Rehabilitation Medicine Training and Research Hospital, ${ }^{2}$ Department of Physical and Rehabilitation Medicine, Ankara Occupational Diseases Hospital, Ankara, ${ }^{3}$ Department of Physical and Rehabilitation Medicine, Dicle University Medical School, Diyarbakır, 4Department of Physical and Rehabilitation Medicine, Hacettepe University Medical School Ankara, Turkey.

Correspondence: Dr İ Batmaz, DicleÜniversitesi Tıp Fakültesi Hastaneleri FTR AD Diyarbakır, Turkey. Fax: 90412 2488537, e-mail: ibrahimbatmaz82@hotmail.com 


\section{INTRODUCTION}

Hydatid cyst disease (HD) is an infestation caused by the larval stage of the tape worm Echinococcus granulosus. It predominantly occurs in liver $(\sim 70 \%)$ and lungs $(\sim 20 \%)$ (1). Bone involvement is quite rare $(0.5 \%-4 \%)$; and among those, spinal disease is the most common type (44\%-50\%). The lesions are usually seen at the thoracic (50\%) and lumbar $(20 \%)$ regions and less at the cervical and sacral levels $(11 \%)(2,3)$.

Concerning the clinical manifestations, spinal involvement may present with various neurological findings (usually due to spinal cord and/or radicular nerve root compression) depending on the area of involvement $(1,2)$. Additionally, it has been previously reported that this benign disease (especially the spinal form) may turn out to display a sort of 'malignant' course because of its high rate of recurrence (4).

Likewise, in this report, we present two patients who had recurrent spinal HD with two different outcomes (neurogenic bladder and paraplegia) despite intense medical treatment and repeat surgeries.

\section{CASE 1}

A 50-year-old male was under follow up with a diagnosis of lumbar HD. His medical history comprised an initial attack of paraparesis (10 years ago) which had completely resolved after L3 vertebra anterior decompression and posterior instrumentation surgery. He had been diagnosed to have HD after surgery at that time. Later on, during his follow up, he had been operated 4 times more (decompression and stabilization) due to his persistent findings of sciatica. Eventually, he was operated for the $6^{\text {th }}$ time (anterior wide decompression and cage implantation) as he developed cauda equina syndrome and paraplegia; however, the neurological findings did not improve. Currently, he suffers painful low back motions and his 
physical examination is consistent with paraplegia. Recent computed tomography displayed spinal HD (Fig. 1).

\section{CASE 2}

A 39-year-old female was hospitalized for neurogenic bladder rehabilitation. Her medical history revealed sacral HD 23 years ago when she had had left-sided sciatica, hypoesthesia and urinary incontinence. She had been operated accordingly and her findings had disappeared. She had also used albendazole for one year. Starting from the end of that year, in total, she had been operated 8 times for sacral HD and 5 times for neurogenic bladder.

Currently, she has painful low back motions, S1 hypoesthesia and she is using intermittent catheterization (8 times daily). A control magnetic resonance imaging showed sacral HD (Fig. 2).

\section{DISCUSSION}

Spinal HD is radiologically categorized as intramedullary, intradural extramedullary, extradural, vertebral and paravertebral hydatid cyst lesions extending to the spinal structures (2, 3). Approximately $90 \%$ of the cases fall into the extradural group, most commonly involving the vertebral body (4). Parasites are considered to reach the highly vascularized centre of the vertebral body through the portal vertebral venous shunts. Cysts then penetrate the trabeculae in the direction of least resistance and initiate a destruction/expansion in the vertebra corpus. Eventually, after the cortex, the cyst invades the extradural space, causing neurologic deficits $(1,3)$. Likewise, in our cases, there was both extradural and paravertebral involvement. 
Initial clinical findings of spinal HD can be nonspecific. Spinal HD may manifest with any symptom due to bone destruction or spinal cord compression, but a long history of back pain and/or subacute symptoms associated to spinal cord/nerve compression (radicular pain, sphincter disturbance, bladder dysfunction, paraparesis, paraplegia) are the most frequent (1). Back pain has been observed in $85 \%$, radicular pain in $25 \%-95 \%$ and paraparesis in $25 \%-77 \%$ of the patients (5). Additionally, neurogenic bladder and bowel dysfunction can be observed with sacral involvement (3). On the other hand, paraplegia is definitely the most serious complication $(1,5)$. In our patients, low back and radicular pain and urinary incontinence were the initial manifestations whereby the clinical scenario comprised neurogenic bladder and paraplegia eventually.

Concerning surgical treatment, it may not be curative in most cases with a recurrence rate of $30-40 \%$. With recurrences presenting many years after initial treatment of spinal HD, the assessment of treatment outcome remains difficult (2). Yet, especially in late cases, spinal surgery is challenging due to concomitant neural and bony involvement (4). As such, adjuvant administration of medications i.e. mebendazole, benzimidazole or albendazole are used to improve the treatment outcome on long-term basis (3). Since the introduction of mebendazole and albendazole, surgery with concomitant and subsequent benzimidazole administration became the widely accepted treatment standard. On the other hand, in advanced cases with extensive vertebral involvement, multiple palliative surgeries together with antiparasitic therapy can prolong survival over many years (2).

In closing, presenting the two patients of ours with major complications, we tried to render that despite every effort (medical/surgical), the outcome might be quite unfavorable in this otherwise benign infectious disease. Therefore, we once again underscore the need for early diagnosis and prompt management especially in endemic regions of HD. 
Disclosures: Financial disclosure statements have been obtained, and no conflicts of interest have been reported by the authors or by any individuals in control of the content of this article. No conflicts of interest to declare

\section{REFERENCES}

1. Neumayr A, Tamarozzi F, Goblirsch S, Blum J, Brunetti E. Spinal cystic echinococcosis-a systematic analysis and review of the literature: part 1. Epidemiology and anatomy. PLoS Negl Trop Dis 2013; 7: e2450. doi: 10.1371/journal.pntd.0002450.

2. Neumayr A, Tamarozzi F, Goblirsch S, Blum J, Brunetti E. Spinal cystic echinococcosis-a systematic analysis and review of the literature: part 2. Treatment, follow-up and outcome. PLoS Negl Trop Dis 2013; 7: e2458. doi: 10.1371/journal.pntd.0002458.

3. Papakonstantinou O, Athanassopoulou A, Passomenos D, Kalogeropoulos I, Balanika A, Baltas C, et al. Recurrent vertebral hydatid disease: spectrum of MR imaging features. Singapore Med J 2011; 52: 440-5.

4. Hamdan TA. Hydatid disease of the spine: a report on nine patients. International Orthopaedics (SICOT) 2012; 36: 427-32.

5. Hemama M, Lasseini A, Rifi L, Boutarbouch M, Derraz S, Ouahabi AE, et al. A sacral hydatid cyst mimicking an anterior sacral meningocele. J Neurosurg Pediatrics 2011; 8: 526-9. 


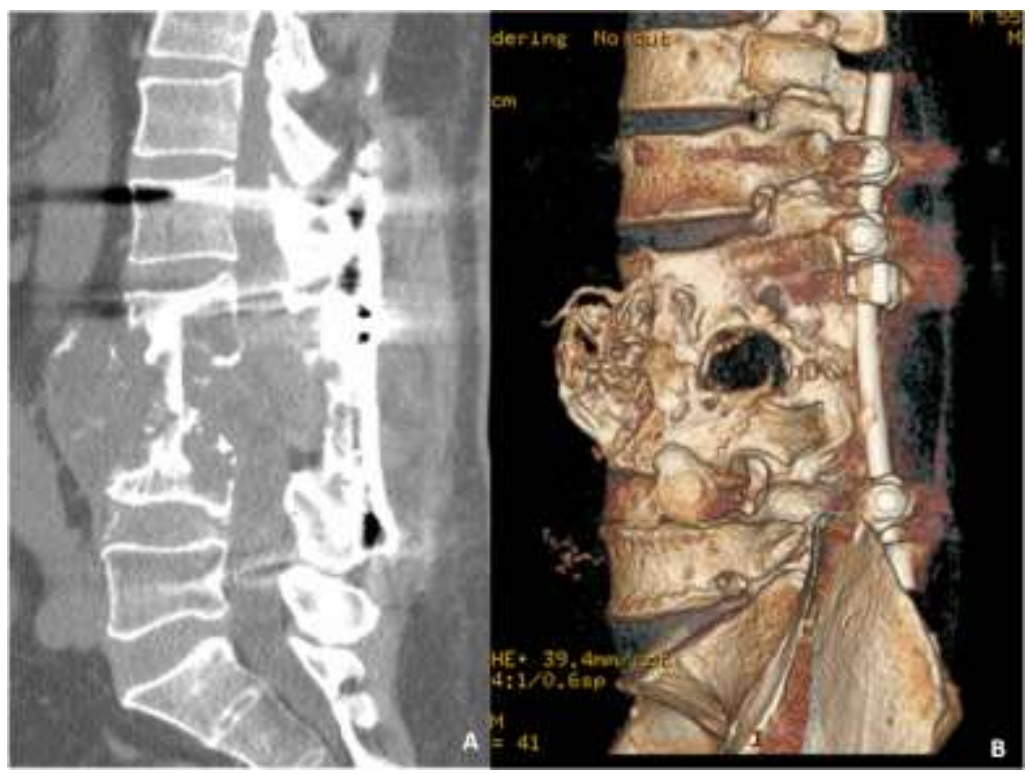

Figure 1: Sagittal reformatted (A) and 3D (B) computed tomography scans outlining the lumbar defect.

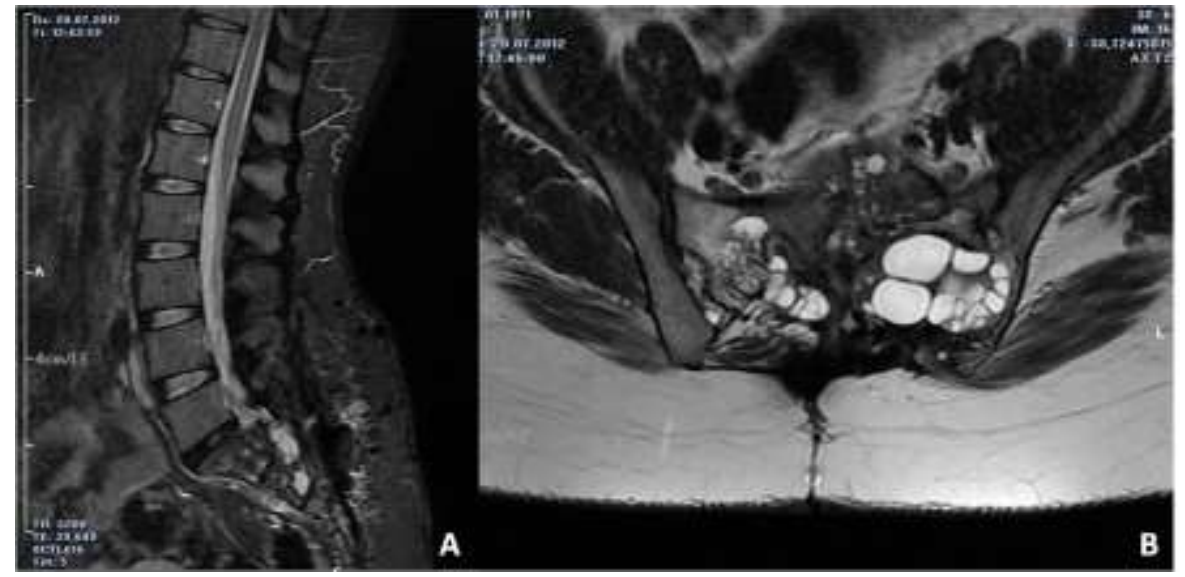

Figure 2: Sagittal (A) and axial (B) T2-weighted images showing massive bone destruction of the sacral vertebrae. 